\title{
Line Emission from Warm Material in NGC 5548
}

\author{
J. S. KAASTRA, ${ }^{1}$ R. MEWE, ${ }^{1}$ AND N. ROOS $^{2}$ \\ ${ }^{1}$ SRON, Sorbonnelaan 2, 3584 CA Utrecht, The Netherlands \\ ${ }^{2}$ Sterrewacht Leiden, Niels Bohrweg 2, 2300 RA Leiden, The Netherlands
}

\begin{abstract}
EUVE observations of the Seyfert 1 galaxy NGC 5548 have shown the presence of line emission features identified as a Ne VII/Ne VIII blend at $88 \AA$ and Si VII emission at $70 \AA$. The lines show significant broadening (FWHM $3800 \mathrm{~km} \mathrm{~s}^{-1}$ ) placing the emitting region at the same distance as the inner broad-line region. A fit to a thermal plasma yields a temperature of $6 \times 10^{5} \mathrm{~K}$. The line emission can be attributed to the warm absorbing material discovered before from oxygen and iron absorption features, which appears to be an optically thin (for the Lyman continuum), highly ionized phase of the broad-line region.
\end{abstract}

\section{Introduction}

NGC 5548 is one of the brightest Seyfert galaxies in the soft X-ray band (BranduardiRaymont et al. 1984). The spectrum and variability of both soft and hard X-rays was studied extensively using Exosat observations by Kaastra \& Barr (1989). The spectrum could be decomposed into a hard power-law component plus a modified-blackbody spectrum which was attributed to direct radiation from the accretion disk. The power-law was identified as disk radiation up-scattered by the inverse Compton process in a hot corona surrounding a part of the disk.

The spectrum of the soft component was highly variable, both in flux and shape, on a 0.5 day time scale. The spectral variations can be explained by correlated temperature luminosity changes of the accretion disk (Kaastra 1991a,b), or alternatively by ionization of a warm absorber in the line of sight. Evidence for a warm absorber in NGC 5548 arises from Ginga observations of an Fe edge around $8 \mathrm{keV}$ (Nandra et al. 1991) and from the discovery by Rosat of an O VII or O VIII edge near $0.8 \mathrm{keV}$ (Nandra et al. 1993). The first $A S C A$ observations (Fabian et al. 1994) confirmed the presence of the O VII and $O$ VIII edge. The equivalent Hydrogen column densities implied are of the order of $10^{26} \mathrm{~m}^{-2}$.

The low-energy spectrum of NGC 5548 is thus rather complicated, containing possible contributions from the accretion disk, the power-law, a warm absorber and galactic absorption. Observations with EUVE provide an excellent opportunity to study this complicated energy band.

\section{EUVE Observations}

Three EUVE observations of NGC 5548 were obtained in the spring of 1993 . These observations have been described in detail elsewhere (Kaastra et al. 1995), but here we summarise them. The longest observation (exposure time $332000 \mathrm{~s}$ ) was obtained between March 10-24, 1993; a second observation with exposure time $226000 \mathrm{~s}$ between April 26 and May 4, 1993; and a short third observation of $71000 \mathrm{~s}$ between May 12-14, 1993.

Especially during the first observation NGC 5548 showed significant line emission. At least two significant line features were visible. The strongest feature at $\sim 88 \AA$ is identified 
as a blend of Ne VII $88.13 \AA$ (transition $2 \mathrm{p}{ }^{3} P_{1}-4 \mathrm{~d}^{3} D_{2,3}$ ) and the Ne VIII $88.10 \AA$ doublet (transition $2 \mathrm{~s}^{2} S_{1 / 2}$ (ground state) $-3 \mathrm{p}^{2} P$ ). The second line at $70.02 \AA$ is a blend of Si VII $\left(2 \mathrm{p}^{4}{ }^{3} \mathrm{P}-2 \mathrm{p}^{3} 3 \mathrm{~d}^{3} D,^{3} \mathrm{P}\right)$. The lines have intrinsic luminosities (corrected for galactic absorption) of $5.1 \times 10^{35} \mathrm{~W}$ and $2.6 \times 10^{35} \mathrm{~W}$, respectively, similar to the average luminosity of e.g., the hydrogen Ly $\alpha$ and C IV $1549 \AA$ lines.

The lines are significantly broadened, with a FWHM of $3800 \pm 1700 \mathrm{~km} \mathrm{~s}^{-1}$. If we interpret this line broadening as due to Doppler shifts corresponding to motion with typical Keplerian velocities around the central black hole, we can derive the distance of the emitting material. From optical/UV monitoring campaigns, the distance scale of the optical broad-line region is reasonably well constrained. The central mass derived from the correlation between the line width and the line lag of the optical/UV lines with respect to the UV continuum is $2.7 \times 10^{7} \mathrm{M}_{\odot}$ (Krolik et al. 1991) or $3.7 \times 10^{7} \mathrm{M}_{\odot}$ (Clavel et al. 1992). We adopt a central mass of $3 \times 10^{7} \mathbf{M}_{\odot}$. Using the FWHM of the $\mathrm{Ne}$ VII/VIII blend $\left(3800 \mathrm{~km} \mathrm{~s}^{-1}\right)$, we derive a distance of $3 \times 10^{14} \mathrm{~m}$, with an uncertainty of a factor of 2 . The plasma emitting the lines observed by $E U V E$ then has a similar distance to the central source as the optical broad-line region.

\section{Emission from a Warm Absorber}

Recently it has been suggested by Netzer (1993) that the warm absorber that appears to be present in many AGN should manifest itself not only by absorption, but also by emission from the warm material.

The EUVE spectrum of NGC 5548 obtained during the first observation period was fitted by a thermal plasma in collisional ionization equilibrium (CIE), using the SPEX code (Kaastra \& Mewe, 1993) which is an extended and updated version of the older code of Mewe et al. (1985). The best fit yields a temperature of $(6.0 \pm 1.4) 10^{5} \mathrm{~K}$, with an emission measure of $2.2 \times 10^{72} \mathrm{~m}^{-3}$. Of course the emitting region needs not to be in CIE, but could as well be in photo-ionization equilibrium (PIE). In the latter case, the temperature will be somewhat lower. Can this emission component be attributed to the warm absorber?

Estimates by Kaastra et al. (1995) (see also section 4) showed that the parameters of the emitting region are entirely consistent with the properties of the warm absorber as deduced from the Ginga, Rosat and $A S C A$ observations.

Other evidence for line emission in Seyfert galaxies obtained from $E U V E$ observations is found in Mkn 478 (Liedahl et al. 1995). In Mkn 478 there is evidence for a blend of Fe-M, Si and Mg emission, in particular near $85 \AA$; the derived emission measure, line broadening and temperature are within a factor of $\sim 2$ consistent with our findings for NGC 5548!

Also recently George et al. (1994) found evidence for O VII and O VIII line emission in the $A S C A$ spectrum of NGC 3783 , indicating emission from a plasma similar to the emitting regions in NGC 5548 and Mkn 478.

Emission from the warm absorber thus seems to be not an unusual feature in Seyfert galaxies.

\section{The Warm Absorber As a Hot Phase of the Broad-Line Region}

Recently Hamann et al. (1995a) studied a sample of $z \sim 1$ quasars with the HST. In several of these sources there was evidence for strong, broad-line emission of the Ne VIII $774 \AA$ line. This line is unvisible in Seyfert galaxies due to the strong absorption by our galaxy, but in high redshift quasars it shifts beyond the Lyman edge and thus becomes 
visible. The line actually is the doublet of the $2 \mathrm{~s}-2 \mathrm{p}$ transition in $\mathrm{Li}$-like neon, while the $88.10 \AA$ line detected by $E U V E$ is the corresponding $2 \mathrm{~s}-3 \mathrm{p}$ transition of the same ion. The luminosity deduced for the Ne VIII $774 \AA$ line is of the same order of magnitude as the luminosity for the C IV $1549 \AA$ line and Ly $\alpha$. The same line is also seen in absorption in other $z \sim 1$ quasars (Hamann et al. 1995b). These authors also suggest that the Ne VIII line emitting material is the same as the warm absorber. Thus the warm absorber might be identified as a highly ionized phase of the broad-line clouds.

Shields et al. (1995) have elaborated this idea further. They conclude that the broadline region consists of at least 3 phases. The low-ionization component, responsible for e.g., the Mg II emission and the high-ionization component responsible for e.g., Ly $\alpha$ and C IV $1549 \AA$ are known for some time. A highly ionized component that is optically thin for the Lyman continuum and fully ionized in hydrogen is new in these models. This component can account for the warm absorber, as the column density and ionization stage are similar.

Shields et al. have modelled the BLR of NGC 5548 by such a multi-phase medium. They used clouds with a column density of $10^{25} \mathrm{~m}^{-2}$, hydrogen density $10^{17} \mathrm{~m}^{-3}$, and microturbulence velocity of the order of $100 \mathrm{~km} \mathrm{~s}^{-1}$. They argue that the covering factor of the optically thin medium is probably high, and since the continuum in NGC 5548 is probably hard enough, emission by the warm absorber is likely. They also suspected that filtering of the intrinsic AGN continuum due to the warm absorber may be important for the radiation field as seen by the clouds at larger radii.

Let us now make some estimates for the Ne VIII $88 \AA$ line of NGC 5548 . In CIE this line contains some $60 \%$ of the total flux of the neon blend. In CIE at $6 \times 10^{5} \mathrm{~K}$ and for cosmic abundances, the optical depth in the continuum $\tau_{c}$ near the line is $0.46 N_{26}$, where $N_{26}$ is the column density of a single cloud in units of $10^{26} \mathrm{~m}^{-2}$ (the warm absorber has typically $N_{26} \sim 1$ ). The optical depth at the line centre $\tau_{\ell}$ is $560 N_{26} / \sqrt{1+20 v_{100}^{2}}$ where $v_{100}$ is the microturbulence velocity of the clouds in units of $100 \mathrm{~km} \mathrm{~s}^{-1}$. Under those PIE conditions where Ne VIII is the most abundant neon ion these optical depths are similar within a factor of $2-3$.

In most cases the line is thus optically thick at its centre. Using random walk arguments for the line photons that are emitted and re-absorbed several times by line emission and absorption, before eventually escaping or being absorbed by the continuum, we find that only line photons produced in the outer fraction $f_{e}=\left(\tau_{c} \tau_{\ell}\right)^{-1 / 2}$ of the absorbing cloud can escape. Assuming a microturbulence velocity of the order of $100 \mathrm{~km} \mathrm{~s}^{-1}$, we approximate $f_{\varepsilon} \sim 0.13 \sqrt{v_{100}} / N_{26}$. Thus the line becomes effectively optical thin for column densities below $10^{25} \mathrm{~m}^{-2}$, and is optically thick otherwise. Contrary, the continuum absorption is only modest. As a result, for $N_{26}$ of order unity, the lines will be suppressed considerably as compared to the continuum. Kaastra et al. (1995) showed that from the observed $E U V E$ spectrum it follows that the line-to-continuum ratio can be suppressed by at most a factor of $\sim 5$. Thus the column density during the first $E U V E$ observation was probably a few times $10^{25} \mathrm{~m}^{-2}$. For smaller values of the microturbulence velocity the column density of the EUV-emitter needs to be much smaller than is consistent with the column density of the warm absorber. Note however that Shields et al. (1995) also required a typical micro-turbulence of $100 \mathrm{~km} \mathrm{~s}^{-1}$ in order to explain the optical/UV line ratio's in NGC 5548!

For the geometry of the source we assume a spherical shell with radius $d$, thickness $\Delta$, filled with a fog of $N$ small clouds with individual radius $r$, Hydrogen density $n$, temperature $T$ and filling factor $f$. For $f=1$ we have a homogeneously filled shell. The total emission measure is $Y$. The total column density of the layer is designated by $N_{\mathrm{H}}$. We use a ratio of electron to Hydrogen density of 1.2. For the distance we adopt the value 
of $310^{14} \mathrm{~m}$, deduced from the line broadening (see section 2). The "observed" emission measure $Y_{\text {obs }}$ as deduced e.g., from our CIE fit is the true emission measure $Y$ multiplied by the fraction $f_{e}$. We use $Y=1.2 n^{2} f 4 \pi d^{2} \Delta, N_{\mathrm{H}}=n f \Delta$, and $f=\frac{4}{3} \pi r^{3} N / 4 \pi d^{2} \Delta$. Inserting the relevant numbers, we find that the typical density of the clouds must be $10^{17} \mathrm{~m}^{-3}$, entirely consistent with the assumptions made for the optical thin high ionized broad-line region component used by Shields et al!

For the Ne VIII $774 \AA$ line, $\tau_{\ell}$ is a factor of 4.5 larger than for the $88.10 \AA$ line; however the continuum optical depth is a factor of 30 smaller for the $774 \AA$ line than for the $88.10 \AA$ line. As a result, $f_{e}$ is 2.5 times larger for the $774 \AA$ line than for the $88.10 \AA$ line. Since in CIE the photon emissivity of the $774 \AA$ line is 190 times larger than for the $88.10 \AA$ line, we expect the effective luminosity of the $774 \AA$ line to be 54 times larger than for the $88.10 \AA$ line (and hence than e.g., the C IV $1549 \AA$ line). Of course in NGC 5548 the $774 \AA$ line is completely blocked by the absorption in our own galaxy. In $z \sim 1$ quasars however, an intervening neutral hydrogen column of only $\sim 10^{21} \mathrm{~m}^{-2}$ would be sufficient to make the apparent flux of the $774 \AA$ line similar to that of the UV lines. That material could be situated anywhere in the line of sight from the quasar host galaxy to the point where the photon energy passes the Lyman limit.

\section{The DS Count Rate}

The count rate obtained during our observations with the Deep Survey instrument with the Lexan/boron filter of $E U V E$ (DS) showed large amplitude variations on a typical time scale of half a day (Marshall 1995), similar to the behaviour as observed by EXOSAT (Kaastra \& Barr 1989). The average count rate during the first observation was about $0.06 \mathrm{c} \mathrm{s}^{-1}$, and about 0.08 counts $\mathrm{s}^{-1}$ during the second observation period.

Our model for the warm emitter predicts a contribution to the DS count rate of only $\sim 0.02 \mathrm{c} \mathrm{s}^{-1}$ in the wavelength range $70-100 \AA$. The predicted contribution to the DS count rate below $70 \AA$ is only $0.002 \mathrm{c} \mathrm{s}^{-1}$. Since the deduced size of the line emitting region is $\sim 11$ light days, the warm emitter is not expected to show rapid variability on a day time scale. It is thus evident that most of the DS flux must be produced by a different, strongly variable soft component, which we assume to be identical to the accretion disk spectrum (cf. Kaastra \& Barr 1989).

It is interesting to note that the model of Kaastra \& Barr for the spectrum of NGC 5548 of March 3, 1986 (a simple power law plus modified blackbody emission from the accretion disk) predicts a DS count rate of $0.065 \mathrm{c} \mathrm{s}^{-1}$. Their model was based upon EXOSAT observations. Since the transmission properties of the EXOSAT Lexan filter and the DS Lexan filter are not too different, we may conclude that the average soft X-ray flux in March 1993 was similar to the average soft X-ray flux in March 1986. In NGC 5548, both the soft- and hard X-ray fluxes (e.g. Kaastra 1991b), and the UV continuum and the hard X-ray flux (Clavel et al. 1992) correlate well. Using these correlations we estimate that the $1450 \AA$ continuum level must have been about $4 \times 10^{-17} \mathrm{Wm}^{-2} \AA^{-1}$. This appears to be only some $30 \%$ higher than the observed UV continuum at that time (cf., Peterson \& Korista 1994).

Finally, our model also predicts strong lines to be present at 50.0, 51.8 and $60.6 \AA$ due to S VII, which must be easily detectable by e.g., the LETGS aboard AXAF.

SRON, the Space Research Organization of the Netherlands is supported financially by NWO, the Netherlands Organization for Scientific Research. 


\section{REFERENCES}

Branduard-Raymont, G., Bell-Burnell, S. J., Kellett, B., Fink, H., Molteni, D., \& MCHARDY, I. 1984, EXOSAT observations of active galactic nuclei. in X-ray and UV emission from active galactic nuclei, ed. W. Brinkmann \& J. Trümper, MPE Report, 184., M.P.I., Garching, 88

Clavel, J., Nandra, K., Makino, F., Pounds, K. A., Reichert, G. A., Urry, C. M., Wamsteker, W., Peracaula-Bosch, M., Stewart, G. C., \& Otani, C. 1992, Correlated hard X-ray and ultraviolet variability in NGC 5548, ApJ, 393, 113

Fabian, A. C., Nandra, K., Brandt, W. N., Hayashida, K., Makino, F., \& Yamauchi, M. 1994, Simultaneous ASCA and Rosat observations of NGC 5548. in New Horizon of X-ray astronomy, ed. F. Makino \& T. Ohashi, Tokyo: Universal Academy Press, 573

George, I. M., Turnen, T. J., \& Netzer, H. 1995, The discovery of an O VII emission line in the ASCA spectrum of the Seyfert galaxy NGC 3783, Astroph. J.L, 438, L67

HamanN, F., Zuo, L., \& TytleR, D. 1995a, Broad Ne VIIIA774 in the HST-FOS snapshot survey ABSNAP, ApJL, in press

Hamann, F., Barlow, T., Beaver, E. A., Burbidge, E. M., Cohen, R. D., JunkkariNEN, V., \& LYONS, R. 1995b, Ne VIII 7774 and time-variable associated absorption in the QSO UM 675, ApJ, in press

KAASTRA, J. S. 1991a, The soft X-ray variability of NGC 5548, A\&A, 249, 70

KAASTRA, J. S. 1991b, Soft X-ray variability and the accretion disk of NGC 5548. in Structure and emission properties of accretion disks, ed. C. Bertout, S. Collin, J-P. Lasota, J. Tran Than Van, IAU Colloq. 129, Gif sur Yvette: Editions Frontières, 449

KAASTRA, J. S. \& BARR, P. 1989, Soft and hard X-ray variability from the accretion disk of NGC 5548, A\&A, 226, 59

KaAstra, J. S. \& Mewe, R. 1993, The Mewe et al., plasma emission code, Legacy, 3, 16

KaAstra, J. S., Roos, N., \& Mewe, R. 1995, EUVE observations of NGC 5548, A\&A, in press

Krolik, J. H., Horne, K., Kallman, T. R., Malkan M. A., \& Edelson, R. A. 1991, Ultraviolet variability of NGC 5548-dynamics of the continuum production region and geometry of the broad-line region, ApJ, 371, 541

Liedahl, D., Paerels, F., Hur, M., Fruscione, A., Kahn, S., \& Bowyer, S. 1995, The EUV spectrum of the Seyfert 1 galaxy Mrk 478, these proceedings

MARShall, H. L. 1995, Variability and spectra of AGN in the EUV and the relation to other bands, these proceedings

Mewe, R. ., Gronenschild, E. H. B. M., \& van Den, Oord, G. H. J. 1985, Calculated $\mathrm{X}$-radiation from optically thin plasmas. V., A\&AS, 62, 197

Nandra, K., Pounds, K. A., Stewart, G. C., George, I. M., \& Hayashida, K. 1991, Compton reflection and the variable X-ray spectrum of NGC 5548, MNRAS, 248, 760

Nandra, K., Fabian, A. C., George, I. M., Branduardi-raymont, G., Lawrence, A., Mason, K. O., Mchardy, I. M., Pounds, K. A., Stewart, G. C., \& Ward, M. J. 1993, A Rosat observation of NGC 5548, MNRAS, 260, 504

NETzER, H. 1993, Ionized absorbers, ionized emitters, and the X-ray spectrum of active galactic nuclei, ApJ, 411, 594

Peterson, B. M., \& Korista, K. T. 1994, Intensive spectroscopic monitoring of NGC 5548 with HST and IUE, in Multi-wavelength continuum emission of AGN, ed. T.J.-L. Courvoisier \& A. Blecha, IAU Symp. 159, Dordrecht: Kluwer, 177

Shields, J. C., Ferland, G. J., \& Peterson, B. M. 1995, Optically thin broad-line clouds in active galactic nuclei, ApJ, 441, 507 